\title{
Manganese-containing bionanocomposites on the basis of natural polysaccharides as novel universal micronutrients for Solanum tuberosum $\mathbf{L}$.
}

\author{
Nozhkina O.A. ${ }^{1}$, Perfileva A.I. ${ }^{1 *}$, Khutsishvili S.S. ${ }^{2}$, Ganenko T.V. ${ }^{2}$ \\ ${ }^{1}$ A.E. Favorsky Irkutsk Institute of Chemistry SB RAS, Irkutsk, 664033, Russia \\ ${ }^{2}$ Siberian Institute of Plant Physiology and Biochemistry SB RAS, Irkutsk, 664033, Russia \\ * email: alla.light@mail.ru
}

The study of the qualitative and quantitative effect of manganese on the vegetation of plants was carried out on the model system of potatoes in vitro, new information was obtained on the mechanism of migration of metal ions, the general toxicological effect of metal on development and the identification of optimal conditions for their entry into the plant organism using the example of Solanum tuberosum L. Novel manganesecontaining bionanocomposites based on polysaccharides (arabinogalactan, sulfated arabinogalactan, $\kappa$-carrageenan) have been synthesized as potential universal trophic low-dose micronutrients, and the structural features of nanocomposites, the ability of original matrices to form stable composite materials and their supramolecular organization have been investigated. The data obtained are relevant in the light of studying the mechanisms of potato survival on saline soils and in private farms in the immediate vicinity of industrial enterprises, and also allow one to assess the plant's capabilities for metal accumulation. The study of the development of plants in nutrient media with different salt content made it possible to compare with plants developing in a nutrient medium with bionanocomposites as a carrier of mineral fertilizers. It is important to note that potato plants grown on a medium with nanocomposites performed better in biometrics. Also, among the most important results it is worth noting that the resulting nanocomposites have an antibacterial effect against the phytopathogenic bacteria Clavibacter sepedonicus and the formation of bacterial biofilms. Growing plants in an environment with manganese-containing nanocomposites promoted an increase in the activity of the protective enzyme peroxidase in leaf tissues as compared to the control. In addition, according to the results obtained, the studied manganesecontaining nanocomposites are safe for representatives of soil microflora.

Acknowledgments: The reported study was funded by RFBR 20-016-00152 A. The authors are grateful to the Baikal Analytical Center of Collective Use, SB RAS. 